\title{
Mother's Own Milk: How Does It Differ from Donor Milk for the Baby
}

\author{
Josef $\mathrm{Neu}$
}

Keywords: mother's own milk, donor milk, human milk

$\mathbf{G}$ UIDELINES FROM the American Academy of Pediatrics recommend that all preterm infants receive mother's own milk or pasteurized donor milk if mother's own milk is unavailable. ${ }^{1}$ However, donor milk lacks sufficient levels of protein and other nutrients (e.g., calcium and phosphorus) to support adequate growth in preterm infants, and fortification is required to meet nutrient requirements. ${ }^{2-4}$ Furthermore, many essential bioactive components of breast milk are lost with standard pasteurization techniques (Table 1). For example, the activity of bile salt-stimulated lipase, an enzyme that facilitates lipid hydrolysis and helps with fat digestion, is completely abolished with pasteurization and is not found in formula. ${ }^{56}$ Similarly, alkaline phosphatase, an enzyme that dephosphorylates lipopolysaccharide to downregulate inflammatory responses, is nearly completely inactivated after pasteurization. ${ }^{7}$ Pasteurization also destroys the majority of live microbes present in donor milk.

Human milk feeding contributes to the development of the infant gut microbiome, yet recent studies have reported the presence of microbes in the placenta, amniotic fluid, and meconium, suggesting that the initial colonization of the infant gut may begin in the womb. ${ }^{8-11}$ The mode of delivery (i.e., cesarean versus vaginal delivery) can also influence development of the early intestinal microbiota and may have long-term consequences. Infants delivered through cesarean section have been shown to have higher odds of several chronic diseases, such as type 1 diabetes, obesity, asthma, and allergies, possibly due to perturbations in the early microbiota. ${ }^{12}$ Initial studies suggest that exposing infants born through cesarean delivery to vaginal fluids at birth can partially restore the microbiota ${ }^{13}$; however, the American College of Obstetricians and Gynecologists recommends against vaginal seeding until better efficacy and safety data are available. ${ }^{14}$ In addition to mode of delivery, the method of feeding (i.e., formula fed or breastfed) has also been shown to impact the gut microbiota, and it is possible human milk could be used to restore the microbiome in infants born through cesarean delivery. ${ }^{15}$
Table 1. EfFect of Holder Pasteurization on the Components of Donor Human Milk
Total lipid

Free fatty acids

Lactose and oligosaccharides

Total protein

Alkaline phosphatase

Bile salt-stimulated lipase

Lactoferrin

Lactoferrin iron binding capacity

$\operatorname{Ig} \mathrm{A}$

IgM

IgG

Lactoperoxidase

Lactoperoxidase activity

Lysozyme

TGF-B

IGF-I

IGF-II

IGFBPs

EGF
Lysozyme activity
NA or $\downarrow 3.5-5.5 \%$
$\uparrow 83 \%$
NA
NA or $4 \%$ reduction
$\downarrow 99 \%$
Abolished
NA or $\downarrow 44-91 \%$
$\downarrow 71 \%$
NA or $\downarrow 20-60 \%$
Abolished
$\downarrow 34 \%$
$\downarrow 82 \%$
$\downarrow 88 \%$
$\downarrow 24-60 \%$
NS or $\downarrow 65-85 \%$
NA
$\downarrow 39 \%$
$\downarrow 10 \%$
$\downarrow 7-19 \%$
NA

EGF, epidermal growth factor; Ig, immunoglobulin; IGF, insulinlike growth factor; IGFBP, insulin-like growth factor-binding protein; NA, not available; TGF, transforming growth factor.

Source: Table courtesy of Dr. Peter Hartmann.

The use of antibiotics in the mother during pregnancy and in preterm infants may also affect development of the infant microbiome. The majority of preterm infants born at $<33$ weeks gestation are treated with ampicillin and gentamicin for at least 48 hours. Antibiotic exposure can result in dysbiosis and may lead to adverse events, such as increased risk of necrotizing enterocolitis. Human milk contains a diverse array of microbes unique to each mother that may be able to overcome dysbiosis in the intestinal tract. ${ }^{16}$ The microbial dose from human milk, assuming an intake of $800 \mathrm{~mL}$ per day, is estimated to be $\sim 10$ to the $7-8$ th power bacterial medium, provided the original work is properly cited. 
cells daily, which is close to the amount used in most probiotic studies. The act of breastfeeding may also influence gut bacteria through the enteromammary pathway, which involves the transfer of antigen-specific antibodies through breast milk. This concept was supported in a study in humans that showed changes in immunomodulatory components in mothers' milk (CD45 leukocytes, macrophages, and tumor necrosis factor- $\alpha$ ) in response to active infection in the nursing infant. ${ }^{17}$ It is important to note that simply providing mothers' milk is not the same as breastfeeding; pumping and freezing mothers' own milk and lack of direct mother-infant contact may diminish some of the beneficial effects of breastfeeding.

There are several techniques that may be useful in increasing the use of mothers' own milk or at least making donor milk more like the original, including stimulation of early lactation, transfaunation, and improved pasteurization methods. Compared with donor milk, mothers' own milk has a higher concentration of most microbes and differs in the relative abundance of bacterial genera. ${ }^{18}$ Mothers' own milk could potentially be used to personalize the microbiota of donor milk. ${ }^{18}$ A recent study demonstrated that incubation of donor milk with $10 \%$ of mothers' own milk for 4-8 hours resulted in microbe levels similar to those seen in mothers' own milk. ${ }^{18}$ Alternative pasteurization techniques, such as ultraviolet $\mathrm{C}$ radiation, can be developed that help preserve the essential bioactive components that are lost in the processing of donor milk. ${ }^{19}$ Because many aspects of donor milk are suboptimal for preterm infants, there is a theoretical rationale for preferring mothers' own milk over banked donor milk; however, well-designed randomized controlled studies are needed to further evaluate the benefits of mothers' own milk and donor milk in preterm infants.

\section{Disclosure Statement}

No competing financial interests exist.

\section{References}

1. American Academy of Pediatrics. Breastfeeding and the use of human milk. Pediatrics 2012;129:e827-e841.

2. Embleton ND, Cooke RJ. Protein requirements in preterm infants: Effect of different levels of protein intake on growth and body composition. Pediatr Res 2005;58:855-860.

3. Ballard O, Morrow AL. Human milk composition: Nutrients and bioactive factors. Pediatr Clin North Am 2013; 60:49-74.

4. Ziegler EE. Human milk and human milk fortifiers. In: Nutritional Care ofPreterm Infants: Scientific Basis and Practical Guidelines, Koletzko B, Uauy R, Poindexter B, eds. Basel, Switzerland: S. Karger AG, 2014.
5. Hamosh M. Lipid metabolism in premature infants. Biol Neonate 1987;52(suppl 1):50-64.

6. Casper C, Carnielli VP, Hascoet JM, et al. rhBSSL improves growth and LCPUFA absorption in preterm infants fed formula or pasteurized breast milk. J Pediatr Gastroenterol Nutr 2014;59:61-69.

7. Bender B. Recombinant human tissue non-specific alkaline phosphatase successfully counteracts lipopolysaccharide induced sepsis in mice. Physiol Res 2015;64:731-738.

8. DiGiulio DB, Romero R, Amogan HP, et al. Microbial prevalence, diversity and abundance in amniotic fluid during preterm labor: A molecular and culture-based investigation. PLoS One 2008;3:e3056.

9. Aagaard K, Ma J, Antony KM, et al. The placenta harbors a unique microbiome. Sci Transl Med 2014;6:237ra65.

10. Ardissone AN, de la Cruz DM, Davis-Richardson AG, et al. Meconium microbiome analysis identifies bacteria correlated with premature birth. PLoS One 2014;9:e90784.

11. Willyard C. Could baby's first bacteria take root before birth? Nature 2018;553:264-266.

12. Neu J, Rushing J. Cesarean versus vaginal delivery: Longterm infant outcomes and the hygiene hypothesis. Clin Perinatol 2011;38:321-331.

13. Dominguez-Bello MG, De Jesus-Laboy KM, Shen N, et al. Partial restoration of the microbiota of cesarean-born infants via vaginal microbial transfer. Nat Med 2016;22:250-253.

14. The American College of Obstetricians and Gynecologists. Vaginal seeding. Committee Opinion No. 725. Obstet Gynecol 2017:130:e274-e278.

15. Azad MB, Konya T, Maughan H, et al. Gut microbiota of healthy Canadian infants: Profiles by mode of delivery and infant diet at 4 months. CMAJ 2013;185:385-394.

16. Hunt KM, Foster JA, Forney LJ, et al. Characterization of the diversity and temporal stability of bacterial communities in human milk. PLoS One 2011;6:e21313.

17. Riskin A, Almog M, Peri R, et al. Changes in immunomodulatory constituents of human milk in response to active infection in the nursing infant. Pediatr Res 2012;71:220-225.

18. Cacho NT, Harrison NA, Parker LA, et al. Personalization of the microbiota of donor human milk with mother's own milk. Front Microbiol 2017;8:1470.

19. Christen L, Lai CT, Hartmann B, et al. Ultraviolet-c irradiation: A novel pasteurization method for donor human milk. PLoS One 2013;8:e68120.

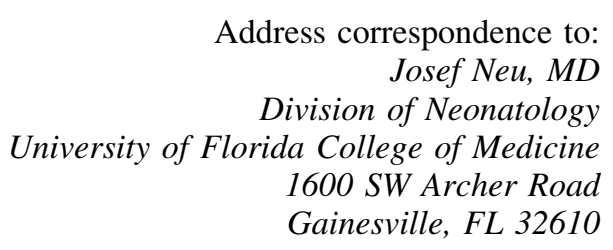

E-mail: neuj@peds.ufl.edu 